\title{
The Magnetosphere of Jupiter: Moving from Discoveries Towards Understanding
}

\author{
Frank Crary \\ University of Colorado, Boulder, Laboratory for Atmospheric and Space Physics \\ (303) 735-2120 \\ frank.crary@lasp.colorado.edu
}

Co-authors:

George B. Clark, Johns Hopkins University, Applied Physics Laboratory

Peter Delamere, University of Alaska

Chuanfei Dong, Princton University

Robert W. Ebert, Southwest Research Institute

Camilla Harris, University of Michigan

George Hospodarsky, The University of Iowa

Sean Hsu, University of Colorado

Hans Huybrighs, European Space Agency

Anna Kotova, L'Institut de Recherche en Astrophysique et Planétologie

Tim Livengood, Goddard Space Flight Center, NASA

Chris Paranicas, Johns Hopkins University, Applied Physics Laboratory

Kurt Retherford, Southwest Research Institute

Elias Roussos, Max Plank Institute for Solar System Research

Yash Sarkango, University of Michigan

Joachim Saur, Universität zu Köln

Jamey R. Szalay, Princeton University

Marissa Vogt, Boston University

Liang Wang, Princton University 


\section{The Magnetosphere of Jupiter: Moving from Discoveries Towards Understanding}

The magnetosphere of Jupiter has been observed by many spacecraft, but most of these results have been discoveries of the global and general properties of the magnetosphere. They have typically raised more questions than they answered. Here we present some of the outstanding questions needed to truly understand Jupiter's magnetosphere and argue that this can be accomplished with small, focused missions.

\section{Scientific questions and unresolved problems:}

Past missions to Jupiter have made great discoveries about its magnetosphere, but for many reasons, those discoveries have raised more questions than they answered. To answer those questions and truly begin to understand this complex system, more observations and observations focused on key issues are necessary. Four open questions and unresolved problems out of a long list are: What is the origin of Jupiter's radiation belts? How and to what extent does the solar wind control of Jupiter's magnetosphere? What is the nature of reconnection at Jupiter and how does it control the dynamics of the magnetotail and magnetospheric boundaries? How does Jupiter's magnetosphere response to the variable volcanic output from Io?

\section{What is the origin of Jupiter's radiation belts:}

Jupiter's radiation belts are by far the most intense in the solar system and have been an enigma since they were discovered over half a century ago [Drake and Hvatum, 1959]. The radiation belts of Jupiter share some similarities with their counterparts at other planets. At the same time, they exhibit significant differences that are key to our understanding of how the basic astrophysical processes of particle acceleration. Several missions to Jupiter, both flyby and orbiter campaigns, have made important observations of Jupiter's plasma sheet region - the region near its magnetic equator. These observations have shown us a host of transport, source and loss processes that are important in forming and sustaining the radiation belts; however, the decades old mystery pertaining to the energetics is still unresolved. A large body of research has been dedicated to the dynamics in the equatorial region, e.g., injections, adiabatic transport, ion-neutral gas interactions, wave-particle interactions, nearly all of the sparse measurements are from the equatorial region. Surveys of the energetic $(>30 \mathrm{keV})$ electron angular distributions by Tomás et al. [2004] and Mauk and Saur [2007] revealed structuring reminiscent of auroral processes in Jupiter's middle magnetosphere. Recent discoveries made by the Juno spacecraft, have put the spotlight on auroral processes as a means to generate high-energy ions and electrons that can fill Jupiter's vast magnetosphere [Mauk et al., 2017; Paranicas et al., 2018], including energetic ion conics [Clark et al., 2017] and upward ion beams [Mauk et al., 2018]. We can reasonably suspect that they must play some role in the generation of energetic particles throughout Jupiter's inner magnetosphere. Unfortunately, Juno can only provide a limited amount of detail of the high-energy electrons (e.g. above the $\sim 1 \mathrm{MeV}$ limit of the Juno/JEDI instrument.) Many mysteries regarding the $>1 \mathrm{MeV}$ electrons still exists and understand their distribution at Jupiter has broad applications to particle acceleration in planetary and astrophysical systems. 


\section{How and to what extent does the solar wind control of Jupiter's magnetosphere?}

It is commonly assumed that Jupiter's magnetospheric dynamics are internally driven, by Jupiter's 10 hour rotation period and the volcanic activity of Io [e.g., Krupp et al., 2004; Vasyliūnas, 1983]. However, the extent to which the solar wind influences the magnetosphere is still under debate [e.g., Cowley et al., 2008; McComas \& Bagenal, 2007], due in part to the lack of an upstream solar wind monitor on Jupiter. The solar wind directly influences the location of the Jovian bow shock and magnetopause (and therefore the size of the magnetosphere), has been shown to influence the intensity and morphology of the auroral radio and ultraviolet emissions, and transports energy and mass into the magnetosphere. Disentangling the Jovian, Io, and solar wind influences is critical in understanding the overall magnetospheric dynamics and especially the auroral morphology. An analysis of Galileo data [Vogt et al., 2019] suggests that activity in Jupiter's magnetosphere is produced by the interplay of internal and external factors with roughly equal significance. We strongly encourage future missions to the outer solar system which include long-term upstream solar wind monitoring and simultaneous observations of Jupiter's magnetosphere and aurora.

\section{What is the nature of reconnection at Jupiter and how does it control and dynamics of the magnetotail and magnetospheric boundaries?}

Magnetic reconnection is an important physical process that allows for the release of mass and energy from a magnetosphere and is a fundamental aspect of a magnetosphere's dynamics. In the Earth's magnetosphere, reconnection at the day side of the magnetopause and in the magnetotail is driven by the solar wind., in a process called the Dungey cycle [Dungey, 1961]. By comparison, at Jupiter, it is commonly assumed that centrifugal stresses are the dominant factor driving magnetospheric dynamics [e.g. Krupp et al., 2004]. The proposed internally-driven tail reconnection process is known as the Vasyliūnas cycle [Vasyliūnas, 1983]. It occurs as mass loaded flux tubes rotate into the night side, are stretched radially by the centrifugal force, break off, and release a plasmoid. Plasmoids are structures which form during reconnection when part of the plasma sheet breaks off, releasing a plasma bubble on closed loops of disconnected field lines that can be ejected down the tail. While the Vasyliunas cycle is generally thought to drive tail reconnection at Jupiter, this picture is almost purely theoretical due to the limited data from Jupiter's magnetotail.

The New Horizons mission, in flying down the magnetotail following its Jupiter flyby, did not observe an organized magnetotail, with an Earth-like current sheet and well defined lobes. Nor did it observe large-scale plasmoids. [McComas et al, 2007] Instead, it observed small, transient "blobs" of plasma, leading to theories of a continuous "drizzle" rather than occasional, large plasmoids [Bagenal, 2007]. Recent measurement by the Juno mission [Vogt et al., 2019] and earlier measurements by Galileo identified [Kronberg et al., 2008; Vogt et al., 2010] suggest an average, down-tail mass flux of under $120 \mathrm{~kg} / \mathrm{s}$, compared to the mass source of over 500 $\mathrm{kg} / \mathrm{s}$ originating from Io. Since this mass is believed to be lost through down-tail flow, the is not clear why the measurements of input and outflow do not match. In both Juno and Galileo data, these transport events occur roughly once every 2-3 days, but the origin of this quasiperiodicity is unknown. 
Reconnection on the day side of Jupiter's magnetosphere also seems different from the same process at Earth. There is little evidence for large-scale reconnection as it is seen at Earth, although this is inconclusive, since there is also little data. However, the role of a viscous interaction along the flanks has been suggested [Delamere and Bagenal, 2013; Masters, 2018]. Earth-like reconnection requires anti-parallel magnetic fields and may be suppressed by some plasma conditions. The appropriate solar wind conditions for reconnection are significantly less common in the outer magnetosphere. The suggested viscous interaction would result from the sheer flow across the magnetopause, on the flanks of the magnetosphere, and drive a KelvinHelmholtz instability. The resulting vortices would drive localized reconnection on small scales. Although not observed at Jupiter, such Kelvin-Helmholtz vortices have been observed on Saturn's magnetopause [Masters et al., 2008].

\section{How does Jupiter's magnetosphere response to the variable volcanic output from Io?}

Io's activity may vary dramatically with short-term volcanic eruptions, or remain roughly constant on a long timescale. As the main source of plasma within the magnetosphere, this implies a similarly dynamic variability in the magnetosphere. The variability of the Io Plasma Torus observed around the Cassini Jupiter flyby suggested that the gas production from Io could change by a factor of 2-3 within a month [Delamere et al., 2004]. Coincidently, the flux of nanodust particles, the so-called "stream particles", increased by 2-3 orders of magnitude during the same period [Krüger et al., 2003]. Stream particles are sub- $\mu \mathrm{m}$ ash particles originated from Io's volcanic plumes that acquired electric charges and are accelerated away from the magnetosphere at speeds comparable to the solar wind. The Hisaki spacecraft has provided a long, continuous data set on EUV emissions from the Io torus [Yoshikawa et al., 2017], but as a small, Earth-orbiting telescope, the spectra are essentially unresolved. Efforts to correlate these and other parts of the system [e.g. Roth et al., 2020] have been inconclusive, since observations of the aurora, Io's atmosphere and Io's volcanic activity are rare (weekly to monthly, or less frequent.) While all these processes are clearly coupled, we know very little about how they are coupled. The data on these various aspects of the Jovian system are often not measured simultaneously, and the sampling is sparse. Characterizing the properties of multiple aspects of the system, over the course of months and daily cadence is critical to understanding the responses of the magnetosphere to the variable volcanic output from its major plasma source - Io.

\section{Past and present exploration of Jupiter's magnetosphere:}

Past missions to Jupiter have collected a wealth of information, but for many reasons the magnetospheric results have been those of discoveries, rather than a deep understanding of this complex system and how its components interact. Many of the past missions have been flybys, and that is an inherent limits when studying a dynamic system. For magnetospheric science, a single flyby can be compared to flying an aircraft from Los Angeles to Boston, measuring quantities like temperature, pressure and wind speed along the route, and then trying to use the data to understand the climate and meteorology of North America. More than flybys are necessary to go beyond the large scale structure and basic properties of a large, complex, and dynamic system. Galileo the first spacecraft to orbit Jupiter, suffered from very low data rates due to the failure of its high gain antenna to deploy. As a result, most of the mission became focused on the satellite encounters. Away from these encounters, the magnetospheric data were very limited (e.g. 
24-s resolution for the magnetometer compared to 32 samples per second for the magnetometer on the Juno spacecraft.) This provided very insightful and tantalizing data, but raised as many questions as it answered. The current Juno mission has an excellent complement of particles and fields instruments, but the instruments and the mission were developed to study the polar magnetosphere and aurora. Due to its polar orbit, the spacecraft orbit spends little time near the equator. The plasma is centrifugally confined to the equator and as a result, so are many dynamically interesting phenomena. Finally, the Europa Clipper and JUICE missions, in development, are very much focused on satellite studies. While they will also make magnetospheric measurements, these are limited by an orbital tour driven by the needs of satellite encounters.

\section{Technical challenges, solutions and lessons from the Earth's magnetosphere:}

Any mission to Jupiter faces considerable challenges, but those are not necessary as great a problem as is often imagined. Magnetospheric missions may be especially capable of coping with many of these problems. Radiation is one of the most infamous problem facing a mission to Jupiter. Fortunately for magnetospheric missions, this harsh radiation environment is confined to the inner magnetosphere. Outside of $\sim 25$ Jovian radii, the environment is not significantly worse than that faced by missions to other planets. While this is no comfort for those involved in missions focused on Jupiter's moons, many of the interesting parts of the magnetosphere are farther from the planet.

Mass, power and telecommunications are concerns common to all missions to Jupiter and do not need to be specifically discussed in this white paper. However it is worth noting that we have over sixty years of experience studying the Earth's magnetosphere. In that time, a huge amount of work has gone into developing good, high heritage instruments which are can be both small and low power. Many approaches to managing data volume and selecting only interesting portions for downlink have also been developed. In addition, the phenomena which require very high resolution, such as microphysics and wave-particle interactions, are basic physical processes. They may manifest themselves in different ways in the different plasma regimes of Earth and Jupiter, but the processes are fundamentally the same. Since these processes can and have been studied at length at the Earth, studying them at Jupiter does not need to drive telemetry requirements.

Another lesson from the Earth's magnetosphere is the extreme value of small spacecraft and multi-spacecraft measurements. Many of the major discoveries concerning the Earth's magnetosphere were not made by large spacecraft with highly sophisticated and resource-intensive instruments. They were made by good, capable instruments which could be sent to the right place. This argues for small spacecraft which often enable getting the right instruments to the right place. Similarly, the experience from the Earth's magnetosphere shows the value of simultaneous measurements from multiple locations. This is often critical to understanding a large, complex system. Even simple measurements like that of the magnetic field may, if made at multiple places and at the same time, be more revealing that a large set of measurements made at only a single location. In light of this, we recommend that NASA consider small, focused missions to study the magnetosphere of Jupiter, and the potential for missions consisting of multiple small spacecraft. In addition, we recommend that, if possible, these missions conducted in collaboration between its 
Planetary Science and Heliophysics Divisions. The Heliophysics Division has both an interest in studying planetary magnetospheres and wealth of talent and experience to contribute. A white paper by Mandt et al. on the subject of inter-division collaborations was submitted to the heliophysics decadal survey, and a similar one is in preparation for the planetary science decadal survey.

\section{Examples of possible, small missions to study Jupiter's magnetosphere}

Many of the open questions critical to our understanding of the Jovian magnetosphere can be addressed by small, focused missions. As noted, experience from decades of work studying the Earth's magnetosphere offers both guidance for making efficient observations and provides many high heritage and low resource instruments. To illustrate this, we will briefly summarize four concepts for small missions focused on key aspects of the Jovian magnetosphere and targeting unanswered questions about it. All these concepts appear to be possible given the resources of a Discovery mission, or less.

One small spacecraft mission, suggested by Clark et al., 2017, would explore the relative contribution to the neutral and plasma tori around Europa from its surface, atmospheric and possible plume interactions as opposed to other Jovian sources. To accomplish this task, there are several key science and measurement objectives that make it possible to distinguish the tori being created from Europa versus Io, the two possible sources being debated in the community. The scientific payload consists of a suite of instruments to diagnose the thermal and suprathermal electron and ion composition and charge states between Io and Europa. This mission will provide the first ever measurements of the detailed ion major and minor species and charge state information in the region between Io and Europa. The high radiation environment in this region had proven too risky for larger, more expensive missions, however it is extremely well suited for lower cost, focused missions. These data are expected to have two additional major impacts on our current knowledge: 1) the data will help us understand the material Io and Europa are pumping into the Jovian system and 2) the data will constrain and confirm or reject the physics driving the chemistry models used to understand the role of Europa and Io in the Jovian system.

The THEMIS (Time History of Events and Macroscale Interactions during Substorms) mission to study the Earth's magnetosphere [Angelopoulos et al., 2008] provides an example of how to understand the dynamics of a planet's magnetotail. This highly successful mission consisted of five small spacecraft, with a mass of $75 \mathrm{~kg}$ each and a carefully selected and focused $25 \mathrm{~kg}$ science payload. The spacecraft were on highly elliptical orbits, spent most of their time near apoapsis, and the orbits were arranged so that, at apoapsis, the spacecraft would be distributed along and across the magnetotail. The multi-point measurements from these spacecraft allowed the mission to track disturbances due to reconnection in magnetotail, as they propagated inward to the Earth and outward to the distant magnetotail. A similar approach would be optimal for understanding the decidedly non-Earthlike magnetosphere and magnetotail of Jupiter. A study of this concept [Crary et al., 2017] considered four small spacecraft and their ability to track flow of mass and energy as they flow from the middle magnetosphere outward and down the magnetotail. Due to the constraints on a mission operating at Jupiter (power, propulsion for orbital insertion and telecommunications) a THEMIS-like spacecraft's mass would grow from the $75 \mathrm{~kg}$ (dry) of 
the THEMIS-like observatories to $540 \mathrm{~kg}$ (wet), however the concept appears to be within the scope and limits of a Discovery mission.

To understand the boundaries of Jupiter' magnetosphere, reconnection and the influence of the solar wind, Ebert et al., 2018, performed a mission concept study as part of NASA's Planetary Science Deep Space SmallSat Studies program. This mission would be a Jupiter-orbiting SmallSat to explore the planet's upstream solar wind environment and magnetospheric boundaries and image its energetic neutral atom (ENA) emissions. Its science objectives are to 1) investigate the impact of the solar wind on Jupiter's magnetosphere, 2) characterize Jupiter's magnetospheric boundaries and 3) determine the contribution from ENAs to mass loss from Jupiter's magnetosphere. These science objectives are met with an instrument payload consisting of a plasma sensor, a magnetometer, and an ENA imager. These goals require an orbit that extends beyond Jupiter's magnetopause and bow shock on the planet's dayside. Mission design is also constrained by the necessity to ride share on a primary spacecraft, preferably until after Jupiter orbit insertion (JOI). After orbital insertion, a series of satellite flybys, mostly with Ganymede, places the spacecraft's apojove at $\sim 150$ RJ from Jupiter on the planet's dayside. The spacecraft design consists of an evolved expendable launch vehicle (EELV) secondary payload adapter (ESPA) compatible frame supporting four triple-deployed solar array panels, a propulsion system, and three science instruments and a vault that houses the majority of the electronics for the spacecraft avionics and payload subsystem.

Even smaller missions could make great contributions to Jovian magnetospheric science. A limit common to many efforts to understand the magnetosphere is the lack of a solar wind monitor. While spacecraft have made measurements of the solar wind while approaching Jupiter, these measurements are typically for a limited period (e.g. one month), and simultaneous measurements of the magnetosphere itself are limited (e.g. to Earth-based measurements of the aurora). Truly understanding the effects of the solar wind on Jupiter's magnetosphere requires simultaneous and continuous measurements of the solar wind and the magnetosphere for a period of many months. This is surprisingly easy to do. Several ideas for such a solar wind monitor have been suggested, and the spacecraft does not even need to enter orbit around Jupiter. One example was suggested by Crary and Bagenal, 2019. On a solar orbit with a aphelion of 5.1 AU the spacecraft could conduct a slow flyby, spending approximately 300 days upstream of Jupiter and within $0.5 \mathrm{AU}$ of the planet. From this position, a magnetometer and a plasma spectrometer could continuously monitor the solar wind while a UV imager could capture hourly images of the planet's at resolution comparable to that of the Hubble Space Telescope's STIS instrument from Earth orbit. Given a launch as a secondary payload or a ride share, a mission of this sort would be possible within the scope of a ESPA-class $(180 \mathrm{~kg}$ ) spacecraft and NASA's SIMPLEx (Small Innovative Missions for Planetary Exploration) program.

\section{Conclusions and recommendations}

Despite the past missions to Jupiter, many questions remain about its magnetosphere and how the different elements of this dynamic and coupled system interact. The past discoveries have shown us how much more there is to learn before we understand Jupiter's magnetosphere. These questions include: What is the origin of Jupiter's radiation belts? How and to what extent does the solar wind control of Jupiter's magnetosphere? What is the nature of reconnection at Jupiter and 
how does it control and dynamics of the magnetotail and magnetospheric boundaries? How does Jupiter's magnetosphere response to the variable volcanic output from Io?

We feel that these unresolved questions can be addressed by future missions, and that small focused missions on scale of Discovery missions (or possibly smaller) can achieve this goal. Priorities include missions to study the magnetotail, the magnetospheric boundaries, the radiation belts and the role the solar wind plays in driving the system's dynamics.

\section{References:}

V. Angelopoulos (2008), Space Sci Rev 141: 5-34

Bagenal, F. (2007), J. Atmos. Sol. Terr. Phys., 69, 387-402

Clark, G., et al., (2017.) Geophysical Research Letters 44, 4419.

Clark, G. et al., (2017) presented at Low Cost Planetary Missions Conference 12

Cowley, S. W. H., et al., (2008). Geophysical Research Letters, 35, L101010.

Crary, F. J., et al. (2017), presented at Low Cost Planetary Missions Conference 12

Crary, F. J. and Bagenal, F. (2019) presented at Interplanetary Small Satellite Conference

Delamere, P.A., Steffl, A., and Bagenal, F., (2004), J. Geophys. Res., 109, A10216.

Delamere, P.A., and Bagenal, F. (2013), J. Geophys. Res., 118, 1-9

Drake, F. D., Hvatum, S. (1959). The Astronomical Journal 64, 329.

Dungey, J. W. (1961), Phys. Rev. Lett., 6, 47-48.

R. W. Ebert, et al., (2018), Proceedings for the 2018 IEEE Aerospace Conference, 2239, 2.

Krüger, H., et al., (2003). Geophys. Res. Lett., 30, 21, 2101.

Kronberg, E. A., J. Woch, N. Krupp, and A. Lagg (2008), J. Geophys. Res., 113, A10202

Krupp, N., et al., in Jupiter: The Planet, Satellites, and Magnetosphere.

Masters, A. (2018). Geophysical Research Letters, 45, 7320-7329

Masters, A., et al., Planetary and Space Science, 57, 1769-1778

Mauk, B. H., Saur, J. (2007.) Journal of Geophysical Research 112, A10221.

Mauk, B. H., et al. (2017.) Geophysical Research Letters 44, 4410.

Mauk, B. H., et al. (2018.) Geophysical Research Letters 45, 1277.

McComas, D. J., and Bagenal, F. (2007). Geophysical Research Letters, 34, L20106.

McComas, D. J., et al., (2007), Science, 318, 2170-220

Paranicas, C., et al. (2018.) Journal of Geophysical Research 123, 1989.

Roth, Lorenz, et al. (2020) Icarus, 350, 113925

Tomás, S, A. et al. (2004). Journal of Geophysical Research (Space Physics) 109, A06203.

Vasyliūnas, V. M. (1983). In Physics of the Jovian magnetosphere, (p. 395).

Vogt, M. F., et al., (2010). J. Geophysical Research, 115, A06219.

Vogt, M. F., et al., (2019). Journal of Geophysical Research, 124, 10171-10199.

Vogt, M. F., et al., (2020). Journal of Geophysical Research, 125, 2019JA027486

Yoshikawa, et al., (2017). Earth Planets Space 69, 110. 